\title{
A Cartesian Compliance Controller for a Manipulator Mounted on a Flexible Structure
}

\author{
Christian Ott, Alin Albu-Schäffer, and Gerd Hirzinger \\ German Aerospace Center (DLR e.V.) \\ Institute of Robotics and Mechatronics \\ 82234 Wessling, Germany \\ Email: christian.ott@dlr.de, alin.albu-schaeffer@dlr.de
}

\begin{abstract}
In this paper the Cartesian compliance control of a manipulator mounted on a flexible base is considered. The proposed control law aims at achieving a desired stiffness and damping in Cartesian coordinates while taking account of the base flexibility. The controller does not use any measurement of the base motion, however a model of the base stiffness is required. For the closed loop system, asymptotic stability in case of free motion is proven. Furthermore, considering interaction tasks, it is shown that the controlled manipulator system has a useful passivity property.
\end{abstract}

\section{INTRODUCTION}

It is well known that the flexibility of the base on which a robot manipulator is mounted can significantly influence the positioning accuracy [1]. Moreover, the base flexibility is also relevant from a stability point of view due to the presence of non-linear couplings between the robot dynamics and the base dynamics.

In many applications it is not possible to measure the motion of the base reliably, but still the controller of the arm should take the base flexibility into account. Otherwise base vibrations and the gravity load of the arm on the flexible base will lead to degraded position accuracy of the end-effector. Typical examples for applications where this is relevant are for instance Micro/Macro-manipulator systems where the macromanipulator represents the base, or mobile manipulation settings where a manipulator is mounted on a mobile base. If the mobile base is actuated by non-rigid wheels, this leads to a considerable elasticity. In order to achieve high position accuracy as required for fine manipulation tasks, one clearly should take this into account.

The control of a robot mounted on a flexible base has been treated by several authors. Nenchev et al. proposed the co-called reaction null-space control [2]. In this method the dynamic redundancy of a kinematically redundant arm is exploited such that the robot can perform its tasks without exciting the vibrations of the base. Furthermore, a gravityfree environment was considered therein. In contrast to that our contribution focuses on a compensation of the static endeffector deviation due to a gravity induced base deflection. In particular it is also applicable both to redundant and to nonredundant manipulators. Therefore, the focus of our paper is somewhat complementary to [2]. Clearly, a combination of the results from [2] with our approach would be possible, and would particularly be useful when the control of a kinematically redundant arm under the effect of gravity is considered.

Another important work on the control of a manipulator mounted on a flexible structure was presented by Ueda and Yoshikawa in [3] where they analyze the robustness of a compliance controller with non-collocated position feedback in a gravity-free environment. Their analysis is based on a modal analysis of the linearized system and includes an additional feedback of the joint acceleration for improving robustness. In contrary to [3], the controller presented in this paper avoids the use of non-collocated feedback and a passivity analysis of the non-linear closed loop system is given.

Several authors treated the vibration damping for a Micro/Macro-manipulator system. In [4] the reaction force of a short rigid manipulator mounted at the tip of a large flexible arm was considered as a control input, and the motion of the short manipulator was commanded such that the reaction force acting on the base produced a damping of the base vibration. Lew and Trudnowski [5] presented a control scheme in which the control torque from a joint level PD-controller is augmented by an additional feedback of the base motion in order to achieve enhanced vibration damping. In [6] this technique was combined with a special filtering of the command. A composite controller was proposed in [7] where the fast part of the control input deals with the joint angle dynamics while the slow part deals with the base motion. This composite controller was used together with an inner loop acceleration feedback.

In this paper we propose a Cartesian compliance controller for a manipulator mounted on a flexible structure. Since the gravity load of the manipulator leads to a deflection of the base, the controller must compensate for this deflection. In particular, it is assumed that the motion of the base cannot be measured but the stiffness of the base is known.

The presented controller is related to our recent results on the control of flexible joint robots [8], [9], [10], [11], [12], [13]. In [8], [9], [12] a passivity based approach for the compliance control of a flexible joint robot was presented. Therein a compensation term for the link side gravity components was computed based only on the motor side positions. This gravity compensation term was combined with a PD-like stiffness 
and damping control law in Cartesian coordinates. In [10] the design was improved by merging the computation of the gravity compensation and the implementation of the stiffness term. In [11] we showed that the used design approach from [10] and [8], [9], [12] is not limited to flexible joint robots but can also be applied to a more general class of systems. In the present paper in particular the results from [10] and [11] are applied and the analysis is refined by the use of the base stiffness as a metric. For simplicity the joints of the robot are assumed non-elastic. An extension to a system with both flexible joints and elastic base, however, is quite straightforward.

The paper is organized as follows. In Section II the controller is formulated and the stability statements are given. The proof of stability is shown in Section III. A simulation study in which the theoretical results are validated is presented in Section IV. Finally, Section V gives a short summary.

\section{Controller Design}

In Section II-A the considered equations of motion and the relevant model assumptions are discussed. Then, in Section II-B the desired closed loop behavior is specified in terms of stiffness and damping. The controller and its stability properties are formulated in Section II-C.

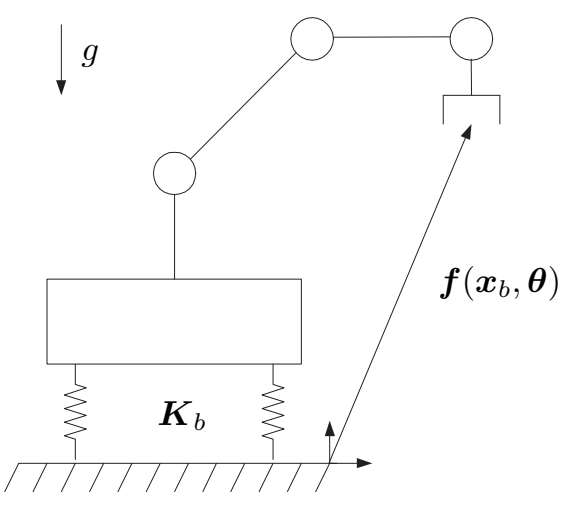

Fig. 1. Manipulator mounted on a flexible base.

\section{A. Equations of motion}

We consider a manipulator arm with $n$ joints mounted on a flexible base with $m$ degrees-of-freedom (DoF), as sketched in Fig. 1. According to the notation used in [2] the joint coordinates of the arm are denoted by $\boldsymbol{\theta} \in \mathbb{R}^{n}$, and the deflection of the base is described by the coordinates $\boldsymbol{x}_{b} \in \mathbb{R}^{m}$. These coordinates are summarized in the vector $\boldsymbol{q}=\left(\boldsymbol{x}_{b}, \boldsymbol{\theta}\right) \in$ $\mathbb{R}^{m+n}$. The considered robot model has the form

$$
\begin{array}{r}
\boldsymbol{M}(\boldsymbol{q})\left[\begin{array}{c}
\ddot{\boldsymbol{x}}_{b} \\
\ddot{\boldsymbol{\theta}}
\end{array}\right]+\boldsymbol{C}(\boldsymbol{q}, \dot{\boldsymbol{q}})\left[\begin{array}{c}
\dot{\boldsymbol{x}}_{b} \\
\dot{\boldsymbol{\theta}}
\end{array}\right]+\left[\begin{array}{c}
\boldsymbol{g}_{b}\left(\boldsymbol{x}_{b}, \boldsymbol{\theta}\right) \\
\boldsymbol{g}_{m}\left(\boldsymbol{x}_{b}, \boldsymbol{\theta}\right)
\end{array}\right]= \\
{\left[\begin{array}{c}
-\boldsymbol{K}_{b} \boldsymbol{x}_{b}-\boldsymbol{D}_{b} \dot{\boldsymbol{x}}_{b} \\
\boldsymbol{\tau}
\end{array}\right]+\boldsymbol{\tau}_{e x t},}
\end{array}
$$

where $\boldsymbol{M}(\boldsymbol{q}) \in \mathbb{R}^{(m+n) \times(m+n)}$ is the inertia matrix of the system. The Coriolis and centrifugal forces are summarized in the term $\boldsymbol{C}(\boldsymbol{q}, \dot{\boldsymbol{q}}) \dot{\boldsymbol{q}}$. The gravity forces on the arm and on the base are defined via a gravity potential function $V_{g}\left(\boldsymbol{x}_{b}, \boldsymbol{\theta}\right)$ as $\boldsymbol{g}_{m}\left(\boldsymbol{x}_{b}, \boldsymbol{\theta}\right)=\left(\partial V_{g}\left(\boldsymbol{x}_{b}, \boldsymbol{\theta}\right) / \partial \boldsymbol{\theta}\right)^{T}$ and $\boldsymbol{g}_{b}\left(\boldsymbol{x}_{b}, \boldsymbol{\theta}\right)=$ $\left(\partial V_{g}\left(\boldsymbol{x}_{b}, \boldsymbol{\theta}\right) / \partial \boldsymbol{x}_{b}\right)^{T}$. The stiffness and damping of the base are described by the symmetric and positive definite matrices $\boldsymbol{K}_{b} \in \mathbb{R}^{m \times m}$ and $\boldsymbol{D}_{b} \in \mathbb{R}^{m \times m}$. The vector $\boldsymbol{\tau} \in \mathbb{R}^{n}$ contains the joint torques of the arm, which are considered as the control inputs. Finally, $\boldsymbol{\tau}_{\text {ext }} \in \mathbb{R}^{m+n}$ is the vector of external torques exerted on the system.

For the stability analysis the following well known property of the system (1) will be needed.

Property 1: For all $\boldsymbol{q} \in \mathbb{R}^{m+n}$ the inertia matrix $\boldsymbol{M ( q )}$ is symmetric and positive definite. For all $\boldsymbol{q}, \dot{\boldsymbol{q}} \in \mathbb{R}^{m+n}$ the matrix $\dot{\boldsymbol{M}}(\boldsymbol{q})-2 \boldsymbol{C}(\boldsymbol{q}, \dot{\boldsymbol{q}})$ is skew symmetric.

In case that the manipulator or the base configuration contains not only rotational but also prismatic joints it is of interest to consider a subset $\mathcal{Q}^{p}$ of $\mathbb{R}^{m+n}$ in which all the prismatic joints are bounded by their respective workspace boundaries. For a robot with rotational joints only one has $\mathcal{Q}^{p}=\mathbb{R}^{m+n}$ instead.

In order to get a formulation which is independent of the chosen physical units for the rotational and translational components of $\boldsymbol{x}_{b}$, particular vector and matrix norms are defined as follows. Let $\boldsymbol{R} \in \mathbb{R}^{m \times m}$ be the square root of the base stiffness matrix $\boldsymbol{K}_{b}$, i.e. $\boldsymbol{K}_{b}=\boldsymbol{R}^{T} \boldsymbol{R}$. Then a vector norm $\|\cdot\|_{K}: \mathbb{R}^{m} \rightarrow \mathbb{R}^{+}$for a vector $\boldsymbol{v} \in \mathbb{R}^{m}$ can be defined via the Euclidean vector norm $\|\cdot\|_{2}$ as

$$
\|\boldsymbol{v}\|_{K}:=\|\boldsymbol{R} \boldsymbol{v}\|_{2}=\left(\boldsymbol{v}^{T} \boldsymbol{K}_{b} \boldsymbol{v}\right)^{1 / 2} .
$$

Corresponding to this vector norm a matrix norm for a matrix $\boldsymbol{A} \in \mathbb{R}^{m \times m}$ is chosen as

$$
\|\boldsymbol{A}\|_{K}:=\left\|\boldsymbol{R}^{-T} \boldsymbol{A} \boldsymbol{R}^{-1}\right\|_{i 2}
$$

Notice that the choice of these norms ensures that the inequality $\left|\boldsymbol{v}^{T} \boldsymbol{A} \boldsymbol{v}\right| \leq\|\boldsymbol{A}\|_{K}\|\boldsymbol{v}\|_{K}^{2}$ holds. Moreover, by construction, the norm of the base stiffness is equal to one, i.e. $\alpha_{k}:=$ $\left\|\boldsymbol{K}_{b}\right\|_{K}=1$.

One further assumption on the system will be needed which ensures that the base stiffness is sufficiently high such that it can withstand the gravity load of the robot. It is well known that in the set $\mathcal{Q}^{p}$ the norm of the Hessian

$$
\boldsymbol{H}_{g}(\boldsymbol{q}):=\frac{\partial^{2} V_{g}\left(\boldsymbol{x}_{b}, \boldsymbol{\theta}\right)}{\partial \boldsymbol{x}_{b}^{2}}
$$

has an upper bound [14]. It will furthermore be assumed that the norm of this gravity Hessian is smaller than the norm of the base stiffness.

Assumption 1: The Hessian $\boldsymbol{H}_{g}(\boldsymbol{q})$ satisfies the condition

$$
\alpha_{g}:=\sup _{\forall \boldsymbol{q} \in \mathcal{Q}^{p}}\left\|\boldsymbol{H}_{g}(\boldsymbol{q})\right\|_{K}<\alpha_{k}=1 .
$$

Clearly, this assumption is not restrictive at all, and usually one has $\alpha_{g}<<1$.

Similar to a flexible joint robot model, the system (1) is underactuated due to the flexibility of the base. For a flexible joint robot each non-actuated DoF is directly connected to the corresponding actuated DoF via the joint stiffness. In (1) the 
non-actuated joints are instead supported by the base stiffness. In the following it is assumed that the deflection of the base cannot be measured, but the stiffness $\boldsymbol{K}_{b}$ of the base is known. For this situation, a Cartesian compliance controller will be designed which compensates for the stationary deflection of the base.

\section{B. Desired Cartesian compliance}

The desired behavior is described in $r \leq n$ Cartesian coordinates $\boldsymbol{f}\left(\boldsymbol{x}_{b}, \boldsymbol{\theta}\right) \in \mathbb{R}^{r}$. The matrices $\boldsymbol{J}_{b}\left(\overline{\boldsymbol{x}}_{b}, \boldsymbol{\theta}\right) \in \mathbb{R}^{r \times m}$ and $\boldsymbol{J}_{m}\left(\boldsymbol{x}_{b}, \boldsymbol{\theta}\right) \in \mathbb{R}^{r \times n}$ are the Jacobian matrices of $\boldsymbol{f}\left(\boldsymbol{x}_{b}, \boldsymbol{\theta}\right)$ with respect to the configuration coordinates $\boldsymbol{\theta}$ and $\boldsymbol{x}_{b}$

$$
\begin{aligned}
\boldsymbol{J}_{b}\left(\boldsymbol{x}_{b}, \boldsymbol{\theta}\right) & :=\frac{\partial \boldsymbol{f}\left(\boldsymbol{x}_{b}, \boldsymbol{\theta}\right)}{\partial \boldsymbol{x}_{b}}, \\
\boldsymbol{J}_{m}\left(\boldsymbol{x}_{b}, \boldsymbol{\theta}\right) & :=\frac{\partial \boldsymbol{f}\left(\boldsymbol{x}_{b}, \boldsymbol{\theta}\right)}{\partial \boldsymbol{\theta}} .
\end{aligned}
$$

The deviation of the Cartesian coordinates from the desired virtual equilibrium position $\boldsymbol{f}_{0} \in \mathbb{R}^{r}$ is denoted by $\boldsymbol{e}\left(\boldsymbol{x}_{b}, \boldsymbol{\theta}\right)=$ $\boldsymbol{f}\left(\boldsymbol{x}_{b}, \boldsymbol{\theta}\right)-\boldsymbol{f}_{0}$. Notice that in this paper we treat only the nonsingular case, i.e. we assume that the manipulator Jacobian $\boldsymbol{J}_{m}\left(\boldsymbol{x}_{b}, \boldsymbol{\theta}\right)$ keeps non-singular. However, the results can readily be combined for instance with the singularity treatment technique from [15].

The desired compliance is then specified by a symmetric and positive definite desired Cartesian stiffness matrix $\boldsymbol{K}_{d} \in$ $\mathbb{R}^{r \times r}$ and a positive definite desired Cartesian damping matrix $\boldsymbol{D}_{d} \in \mathbb{R}^{r \times r}$. Suppose that the external torque $\boldsymbol{\tau}_{\text {ext }}$ is exerted on the robot via a (generalized) constant end-effector force $\boldsymbol{F}_{\text {ext }}$ such that the relation

$$
\boldsymbol{\tau}_{e x t}=\left[\begin{array}{ll}
\boldsymbol{J}_{b}^{T}\left(\boldsymbol{x}_{b}, \boldsymbol{\theta}\right) & \boldsymbol{J}_{m}^{T}\left(\boldsymbol{x}_{b}, \boldsymbol{\theta}\right)
\end{array}\right] \boldsymbol{F}_{\text {ext }}
$$

holds. Then, the steady state of the closed loop system should satisfy the condition

$$
\boldsymbol{F}_{\text {ext }}=\boldsymbol{K}_{d} \boldsymbol{e}\left(\boldsymbol{x}_{b}, \boldsymbol{\theta}\right)
$$

according to the desired stiffness $\boldsymbol{K}_{d}$. Furthermore, the desired Cartesian potential $V_{c}\left(\boldsymbol{x}_{b}, \boldsymbol{\theta}\right)$ consistent with the stiffness matrix $\boldsymbol{K}_{d}$ is given by

$$
V_{c}\left(\boldsymbol{x}_{b}, \boldsymbol{\theta}\right):=\frac{1}{2} \boldsymbol{e}\left(\boldsymbol{x}_{b}, \boldsymbol{\theta}\right)^{T} \boldsymbol{K}_{d} \boldsymbol{e}\left(\boldsymbol{x}_{b}, \boldsymbol{\theta}\right) .
$$

\section{Controller formulation}

Considering (2) any steady state of the system (1) must satisfy the equilibrium conditions

$$
\begin{aligned}
\boldsymbol{g}_{b}\left(\boldsymbol{x}_{b}, \boldsymbol{\theta}\right) & =-\boldsymbol{K}_{b} \boldsymbol{x}_{b}+\boldsymbol{J}_{b}^{T}\left(\boldsymbol{x}_{b}, \boldsymbol{\theta}\right) \boldsymbol{F}_{\text {ext }}, \\
\boldsymbol{g}_{m}\left(\boldsymbol{x}_{b}, \boldsymbol{\theta}\right) & =\boldsymbol{\tau}+\boldsymbol{J}_{m}^{T}\left(\boldsymbol{x}_{b}, \boldsymbol{\theta}\right) \boldsymbol{F}_{\text {ext }} .
\end{aligned}
$$

Notice that equation (6) suggests a feedback law of the form $\boldsymbol{\tau}=\boldsymbol{g}_{m}\left(\boldsymbol{x}_{b}, \boldsymbol{\theta}\right)-\boldsymbol{J}_{m}^{T}\left(\boldsymbol{x}_{b}, \boldsymbol{\theta}\right) \boldsymbol{K}_{d} \boldsymbol{e}\left(\boldsymbol{x}_{b}, \boldsymbol{\theta}\right)$ for gravity compensation and stiffness implementation. But this feedback law is not valid for the considered setting because it is assumed that the base deflection cannot be measured. Instead, one can use (5) to obtain a quasi-static estimate $\overline{\boldsymbol{x}}_{b}(\boldsymbol{\theta})$ of $\boldsymbol{x}_{b}$, which can be used for the controller design. In the following it will be shown how such an estimate can be computed and how its use instead of $\boldsymbol{x}_{b}$ can be considered in the stability proof. According to the desired stiffness relation (3) the controller must ensure that in any steady state the condition

$$
\boldsymbol{g}_{b}\left(\boldsymbol{x}_{b}, \boldsymbol{\theta}\right)=-\boldsymbol{K}_{b} \boldsymbol{x}_{b}+\boldsymbol{J}_{b}^{T}\left(\boldsymbol{x}_{b}, \boldsymbol{\theta}\right) \boldsymbol{K}_{d} \boldsymbol{e}\left(\boldsymbol{x}_{b}, \boldsymbol{\theta}\right)
$$

holds. Equation (7) can be reformulated as

$$
\begin{aligned}
\boldsymbol{x}_{b} & =\boldsymbol{K}_{b}^{-1}\left(-\boldsymbol{g}_{b}\left(\boldsymbol{x}_{b}, \boldsymbol{\theta}\right)+\boldsymbol{J}_{b}^{T}\left(\boldsymbol{x}_{b}, \boldsymbol{\theta}\right) \boldsymbol{K}_{d} \boldsymbol{e}\left(\boldsymbol{x}_{b}, \boldsymbol{\theta}\right)\right) \\
& :=\boldsymbol{T}\left(\boldsymbol{x}_{b}, \boldsymbol{\theta}\right) .
\end{aligned}
$$

For any fixed $\boldsymbol{\theta}$ this can be regarded as an implicit equation for $\boldsymbol{x}_{b}$. Its solution $\overline{\boldsymbol{x}}_{b}(\boldsymbol{\theta})$ can be used as a quasi-static estimate of $\boldsymbol{x}_{b}$.

Notice that Assumption 1 ensures that for $\boldsymbol{K}_{d}=\mathbf{0}$ the function $\boldsymbol{T}\left(\boldsymbol{x}_{b}, \boldsymbol{\theta}\right)$, considered as a mapping $\boldsymbol{x}_{b} \rightarrow \boldsymbol{T}\left(\boldsymbol{x}_{b}, \boldsymbol{\theta}\right)$, is a contraction ${ }^{1}$. Let $\boldsymbol{H}_{c}(\boldsymbol{q})$ be the Hessian ${ }^{2}$ of the Cartesian potential $V_{c}\left(\boldsymbol{x}_{b}, \boldsymbol{\theta}\right)$ w.r.t. $\boldsymbol{x}_{b}$, i.e. $\boldsymbol{H}_{c}(\boldsymbol{q}):=\frac{\partial^{2} V_{c}\left(\boldsymbol{x}_{b}, \boldsymbol{\theta}\right)}{\partial \boldsymbol{x}_{b}^{2}}$. The following assumption then ensures the existence and uniqueness of $\overline{\boldsymbol{x}}_{b}(\boldsymbol{\theta})$.

Assumption 2: There exists a $\rho \in \mathbb{R}$ for which the inequalities $0<\rho<\left(1-\alpha_{g}\right)$ and

$$
\alpha_{c}=\sup _{\forall \boldsymbol{q} \in \mathcal{Q}^{p}}\left\|\boldsymbol{H}_{c}(\boldsymbol{q})\right\|_{K}<\rho .
$$

hold.

One can show that under the Assumptions 1 and 2 the function $\boldsymbol{T}\left(\boldsymbol{x}_{b}, \boldsymbol{\theta}\right)$ is a contraction mapping also for $\boldsymbol{K}_{d} \neq \mathbf{0}$. From this one can follow that the equation $\boldsymbol{x}_{b}=\boldsymbol{T}\left(\boldsymbol{x}_{b}, \boldsymbol{\theta}\right)$ has a unique solution $\boldsymbol{x}_{b}=\overline{\boldsymbol{x}}_{b}(\boldsymbol{\theta})$. Moreover, the iteration

$$
\boldsymbol{x}_{k+1}=\boldsymbol{T}\left(\boldsymbol{x}_{k}, \boldsymbol{\theta}\right)
$$

asymptotically converges to this solution, since $\boldsymbol{T}\left(\boldsymbol{x}_{b}, \boldsymbol{\theta}\right)$ is a contraction [16].

Assumption 2 can be interpreted as an implicit condition on the desired stiffness, with regard to the base stiffness. Loosely speaking it is assumed that the base stiffness $\boldsymbol{K}_{b}$ is sufficiently high such that it can balance the gravity load as well as the effects of the Cartesian stiffness $\boldsymbol{K}_{d}$ on the base coordinates.

Based on the function $\overline{\boldsymbol{x}}_{b}(\boldsymbol{\theta})$ we choose the control law as the sum of a gravity compensation component, a stiffness term, and a damping term in the form

$$
\begin{aligned}
\boldsymbol{\tau} & =\boldsymbol{\tau}_{g}+\boldsymbol{\tau}_{c}+\boldsymbol{\tau}_{d} \\
\boldsymbol{\tau}_{g} & =\boldsymbol{g}_{m}\left(\overline{\boldsymbol{x}}_{b}(\boldsymbol{\theta}), \boldsymbol{\theta}\right) \\
\boldsymbol{\tau}_{c} & =-\boldsymbol{J}_{m}^{T}\left(\overline{\boldsymbol{x}}_{b}(\boldsymbol{\theta}), \boldsymbol{\theta}\right) \boldsymbol{K}_{d} \boldsymbol{e}\left(\overline{\boldsymbol{x}}_{b}(\boldsymbol{\theta}), \boldsymbol{\theta}\right) \\
\boldsymbol{\tau}_{d} & =-\boldsymbol{D}(\boldsymbol{\theta}) \dot{\boldsymbol{\theta}}
\end{aligned}
$$

where the positive definite damping matrix $\boldsymbol{D}(\boldsymbol{\theta})$ is given by

$$
\boldsymbol{D}(\boldsymbol{\theta})=\boldsymbol{J}_{m}^{T}\left(\overline{\boldsymbol{x}}_{b}(\boldsymbol{\theta}), \boldsymbol{\theta}\right) \boldsymbol{D}_{d} \boldsymbol{J}_{m}\left(\overline{\boldsymbol{x}}_{b}(\boldsymbol{\theta}), \boldsymbol{\theta}\right) .
$$

${ }^{1}$ A mapping $T: V \rightarrow V$ defined on a Banach space $V$ with norm $\|\cdot\|$ is called a contraction if there exists a $\rho<1$ such that $\left\|T\left(v_{1}\right)-T\left(v_{2}\right)\right\| \leq$ $\rho\left\|v_{1}-v_{2}\right\|$ holds for all $v_{1}, v_{2} \in V$. More details on contractions can be found, e.g., in [16].

${ }^{2}$ Notice that this Hessian corresponds to the projection of the desired Cartesian stiffness $\boldsymbol{K}_{d}$ via the conservative congruence transformation [17] onto the base coordinates. 
The stability properties of this control law are summarized in the following proposition.

Proposition 1: Consider the system (1) together with the control law (10-13). Under Assumptions 1 and 2 the following properties of the closed loop system hold.

- Desired Stiffness Relation: In case that the external torque $\tau_{\text {ext }}$ is exerted on the robot in form of a constant Cartesian force $\boldsymbol{F}_{\text {ext }}$, then the points defined by the equations $\boldsymbol{F}_{\text {ext }}=\boldsymbol{K}_{d} \boldsymbol{e}\left(\boldsymbol{x}_{b}, \boldsymbol{\theta}\right)$ and $\boldsymbol{x}_{b}=\overline{\boldsymbol{x}}_{b}(\boldsymbol{\theta})$ are equilibrium points of the closed loop system.

- Passivity: For interaction tasks with $\boldsymbol{\tau}_{\text {ext }} \neq \mathbf{0}$ the closed loop system is a passive ${ }^{3}$ mapping from the input $\tau_{\text {ext }}$ to the output $\dot{\boldsymbol{q}}$. This holds even in the redundant case.

- Stability: Consider a non-redundant arm where the number of actuated joints is equal to the number of task coordinates $n=r$. Under the assumption that the Jacobian $\mathrm{d} \boldsymbol{e}\left(\overline{\boldsymbol{x}}_{b}(\boldsymbol{\theta}), \boldsymbol{\theta}\right) / \mathrm{d} \boldsymbol{\theta}$ is nonsingular, the closed loop system for the case of free motion $\left(\boldsymbol{\tau}_{\text {ext }}=\mathbf{0}\right)$ is asymptotically stable.

The proofs of these statements will be given in Section III. But beforehand a short discussion of the control law from an energy based perspective is presented.

\section{An energy based perspective on the control law}

The desired steady state condition (7) was the basis of the controller design. Notice that this equation contains three terms which all can be written as the differential of a potential function with respect to $\boldsymbol{x}_{b}$. In particular, one has

$$
\begin{aligned}
\boldsymbol{g}_{b}\left(\boldsymbol{x}_{b}, \boldsymbol{\theta}\right) & =\left(\frac{\partial V_{g}\left(\boldsymbol{x}_{b}, \boldsymbol{\theta}\right)}{\partial \boldsymbol{x}_{b}}\right)^{T}, \\
\boldsymbol{K}_{b} \boldsymbol{x}_{b} & =\left(\frac{\partial V_{k}\left(\boldsymbol{x}_{b}\right)}{\partial \boldsymbol{x}_{b}}\right)^{T}, \\
\boldsymbol{J}_{b}^{T}\left(\boldsymbol{x}_{b}, \boldsymbol{\theta}\right) \boldsymbol{K}_{d} \boldsymbol{e}\left(\boldsymbol{x}_{b}, \boldsymbol{\theta}\right) & =\left(\frac{\partial V_{c}\left(\boldsymbol{x}_{b}, \boldsymbol{\theta}\right)}{\partial \boldsymbol{x}_{b}}\right)^{T},
\end{aligned}
$$

with the gravity potential $V_{g}\left(\boldsymbol{x}_{b}, \boldsymbol{\theta}\right)$, the stiffness potential $V_{k}\left(\boldsymbol{x}_{b}\right):=1 / 2 \boldsymbol{x}_{b}^{T} \boldsymbol{K}_{b} \boldsymbol{x}_{b}$, and the Cartesian potential $V_{c}\left(\boldsymbol{x}_{b}, \boldsymbol{\theta}\right)$ from (4). By defining the potential function $V_{0}\left(\boldsymbol{x}_{b}, \boldsymbol{\theta}\right)$ as

$$
V_{0}\left(\boldsymbol{x}_{b}, \boldsymbol{\theta}\right)=V_{k}\left(\boldsymbol{x}_{b}\right)+V_{g}\left(\boldsymbol{x}_{b}, \boldsymbol{\theta}\right)-V_{c}\left(\boldsymbol{x}_{b}, \boldsymbol{\theta}\right),
$$

one can, one the one hand, write (7) in the form

$$
\frac{\partial V_{0}\left(\boldsymbol{x}_{b}, \boldsymbol{\theta}\right)}{\partial \boldsymbol{x}_{b}}=\mathbf{0}
$$

On the other hand, the gravity compensation $\tau_{g}$ and the stiffness term $\tau_{c}$ from (10) can be written as $\tau_{g}+\tau_{c}=$

\footnotetext{
${ }^{3}$ A system $\dot{\boldsymbol{x}}=\boldsymbol{f}(\boldsymbol{x}, \boldsymbol{u}), \boldsymbol{y}=\boldsymbol{y}(\boldsymbol{x}, \boldsymbol{u})$ with state $\boldsymbol{x} \in \mathbb{R}^{n}$, input $\boldsymbol{u} \in \mathbb{R}^{m}$ and output $\boldsymbol{y} \in \mathbb{R}^{m}$ is said to be passive, if for any admissible input $\boldsymbol{u}(t)$ the energy that can be extracted from the system in an arbitrary time interval $\left[t_{0}, t_{1}\right]$ is bounded from below [18]: $\exists c \in \mathbb{R}: \int_{t_{0}}^{t_{1}} \boldsymbol{u}(t)^{T} \boldsymbol{y}(t) d t \geq c$. A sufficient condition therefore is given by the existence of a continuous function $S(\boldsymbol{x})$ which is bounded from below and for which the derivative with respect to time along the solutions of the system satisfies the inequality $\dot{S}(\boldsymbol{x})=\frac{\partial S(\boldsymbol{x})}{\partial \boldsymbol{x}} \boldsymbol{f}(\boldsymbol{x}, \boldsymbol{u}) \leq \boldsymbol{u}^{T} \boldsymbol{y}$.
}

$\left(\partial V_{0}\left(\boldsymbol{x}_{b}, \boldsymbol{\theta}\right) / \partial \boldsymbol{\theta}\right)_{\boldsymbol{x}_{b}=\overline{\boldsymbol{x}}_{b}(\boldsymbol{\theta})}^{T}$. Moreover, since $\overline{\boldsymbol{x}}_{b}(\boldsymbol{\theta})$ fulfills (7) this can also be formulated as

$$
\boldsymbol{\tau}_{g}+\boldsymbol{\tau}_{c}=\left(\frac{\mathrm{d} V_{0}\left(\overline{\boldsymbol{x}}_{b}(\boldsymbol{\theta}), \boldsymbol{\theta}\right)}{\mathrm{d} \boldsymbol{\theta}}\right)^{T}
$$

and thus $-V_{0}\left(\overline{\boldsymbol{x}}_{b}(\boldsymbol{\theta}), \boldsymbol{\theta}\right)$ may serve as a potential function for the control input $\tau$.

Finally, for the passivity proof also the Hessian $\boldsymbol{H}_{0}\left(\boldsymbol{x}_{b}, \boldsymbol{\theta}\right):=\frac{\partial^{2} V_{0}\left(\boldsymbol{x}_{b}, \boldsymbol{\theta}\right)}{\partial \boldsymbol{x}_{b}^{2}}$ will be of interest. This Hessian is given by

$$
\boldsymbol{H}_{0}\left(\boldsymbol{x}_{b}, \boldsymbol{\theta}\right)=\boldsymbol{K}_{b}+\boldsymbol{H}_{g}\left(\boldsymbol{x}_{b}, \boldsymbol{\theta}\right)+\boldsymbol{H}_{c}\left(\boldsymbol{x}_{b}, \boldsymbol{\theta}\right),
$$

and thus from Assumption 1 and 2 it follows that the minimum eigenvalue of the matrix $\boldsymbol{R}^{-T} \boldsymbol{H}_{0}\left(\boldsymbol{x}_{b}, \boldsymbol{\theta}\right) \boldsymbol{R}^{-1}$ is bounded from below by a positive number, i.e.

$$
\lambda_{0}>1-\alpha_{g}-\alpha_{c}>0,
$$

where $\lambda_{0}:=\inf _{\forall \boldsymbol{q} \in \mathcal{Q}^{p}} \lambda_{\min }\left(\boldsymbol{R}^{-T} \boldsymbol{H}_{0}\left(\boldsymbol{x}_{b}, \boldsymbol{\theta}\right) \boldsymbol{R}^{-1}\right)$ and $\lambda_{\min }(\boldsymbol{A})$ denotes the minimum eigenvalue of a matrix $\boldsymbol{A}$. Therefore, the function $V_{0}\left(\boldsymbol{x}_{b}, \boldsymbol{\theta}\right)$ is positive definite w.r.t $\boldsymbol{x}_{b}$.

\section{PRoof of Proposition 1}

\section{A. Equilibrium points}

Considering (5)-(6) and (10-13), one can see that the equilibrium equations of the system are given by

$$
\begin{aligned}
\boldsymbol{g}_{b}\left(\boldsymbol{x}_{b}, \boldsymbol{\theta}\right)= & -\boldsymbol{K}_{b} \boldsymbol{x}_{b}+\boldsymbol{J}_{b}^{T}\left(\boldsymbol{x}_{b}, \boldsymbol{\theta}\right) \boldsymbol{F}_{e x t}, \\
\boldsymbol{g}_{m}\left(\boldsymbol{x}_{b}, \boldsymbol{\theta}\right)= & \boldsymbol{g}_{m}\left(\overline{\boldsymbol{x}}_{b}(\boldsymbol{\theta}), \boldsymbol{\theta}\right)+\boldsymbol{J}_{m}^{T}\left(\boldsymbol{x}_{b}, \boldsymbol{\theta}\right) \boldsymbol{F}_{e x t} \\
& -\boldsymbol{J}_{m}^{T}\left(\overline{\boldsymbol{x}}_{b}(\boldsymbol{\theta}), \boldsymbol{\theta}\right) \boldsymbol{K}_{d} \boldsymbol{e}\left(\overline{\boldsymbol{x}}_{b}(\boldsymbol{\theta}), \boldsymbol{\theta}\right) .
\end{aligned}
$$

It can easily be verified that these equations are fulfilled by all points $\left(\boldsymbol{x}_{b}, \boldsymbol{\theta}\right)$ for which

$$
\begin{aligned}
\boldsymbol{e}\left(\overline{\boldsymbol{x}}_{b}(\boldsymbol{\theta}), \boldsymbol{\theta}\right) & =\boldsymbol{K}_{d}^{-1} \boldsymbol{F}_{e x t} \\
\boldsymbol{x}_{b} & =\overline{\boldsymbol{x}}_{b}(\boldsymbol{\theta})
\end{aligned}
$$

hold. Thus, the first property from Proposition 1 is shown.

Next, the passivity statement is considered. Therefore, the potential function $V_{0}\left(\boldsymbol{x}_{b}, \boldsymbol{\theta}\right)$ will be used in order to construct a pseudo energy function $V(\boldsymbol{q}, \dot{\boldsymbol{q}})$. This function will serve as a storage function for the proof of passivity and also as a Lyapunov function for the stability proof in the non-redundant case. Notice that, apart from Section III-E, the results of this paper are valid also for the non-redundant case.

\section{B. Pseudo energy function}

The pseudo energy function $V(\boldsymbol{q}, \dot{\boldsymbol{q}})$ is chosen as the sum of the kinetic energy $V_{k i n}(\boldsymbol{q}, \dot{\boldsymbol{q}})$ of the manipulator and a potential function $V_{\text {pot }}(\boldsymbol{q})$, i.e.

$$
V(\boldsymbol{q}, \dot{\boldsymbol{q}})=V_{k i n}(\boldsymbol{q}, \dot{\boldsymbol{q}})+V_{p o t}\left(\boldsymbol{x}_{b}, \boldsymbol{\theta}\right) .
$$

The kinetic energy is given by

$$
V_{k i n}(\boldsymbol{q}, \dot{\boldsymbol{q}}):=\frac{1}{2}\left[\begin{array}{c}
\dot{\boldsymbol{x}}_{b} \\
\dot{\boldsymbol{\theta}}
\end{array}\right]^{T} \boldsymbol{M}(\boldsymbol{q})\left[\begin{array}{c}
\dot{\boldsymbol{x}}_{b} \\
\dot{\boldsymbol{\theta}}
\end{array}\right]
$$

and clearly is positive definite with respect to the velocities. The potential function $V_{\text {pot }}\left(\boldsymbol{x}_{b}, \boldsymbol{\theta}\right)$ is chosen as the sum of the 
gravity energy, the potential energy of the base flexibility, and the potential function of the control torque

$$
V_{p o t}\left(\boldsymbol{x}_{b}, \boldsymbol{\theta}\right):=V_{k}\left(\boldsymbol{x}_{b}\right)+V_{g}\left(\boldsymbol{x}_{b}, \boldsymbol{\theta}\right)-V_{0}\left(\overline{\boldsymbol{x}}_{b}(\boldsymbol{\theta}), \boldsymbol{\theta}\right) .
$$

By comparison with (15) one can see that $V_{\text {pot }}\left(\boldsymbol{x}_{b}, \boldsymbol{\theta}\right)$ can also be written as

$$
V_{p o t}\left(\boldsymbol{x}_{b}, \boldsymbol{\theta}\right)=V_{0}\left(\boldsymbol{x}_{b}, \boldsymbol{\theta}\right)-V_{0}\left(\overline{\boldsymbol{x}}_{b}(\boldsymbol{\theta}), \boldsymbol{\theta}\right)+V_{c}\left(\boldsymbol{x}_{b}, \boldsymbol{\theta}\right) .
$$

\section{Properties of the potential function}

In the following it is shown that the potential function $V_{\text {pot }}\left(\boldsymbol{x}_{b}, \boldsymbol{\theta}\right)$ is non-negative and even positive definite with respect to $\boldsymbol{e}\left(\boldsymbol{x}_{b}, \boldsymbol{\theta}\right)$ and $\tilde{\boldsymbol{x}}_{b}:=\boldsymbol{x}_{b}-\overline{\boldsymbol{x}}_{b}(\boldsymbol{\theta})$. Therefore, the difference $V_{0}\left(\boldsymbol{x}_{b}, \boldsymbol{\theta}\right)-V_{0}\left(\overline{\boldsymbol{x}}_{b}(\boldsymbol{\theta}), \boldsymbol{\theta}\right)$ must be shown to be positive definite in $\tilde{\boldsymbol{x}}_{b}$. Since $\left(\frac{\partial V_{0}\left(\boldsymbol{x}_{b}, \boldsymbol{\theta}\right)}{\partial \boldsymbol{x}_{b}}\right)_{\boldsymbol{x}_{b}=\overline{\boldsymbol{x}}_{b}(\boldsymbol{\theta})}=\mathbf{0}$, this difference can be written as

$V_{0}\left(\boldsymbol{x}_{b}, \boldsymbol{\theta}\right)-V_{0}\left(\overline{\boldsymbol{x}}_{b}(\boldsymbol{\theta}), \boldsymbol{\theta}\right)=\int_{\overline{\boldsymbol{x}}_{b}(\boldsymbol{\theta})}^{\boldsymbol{x}_{b}} \int_{\overline{\boldsymbol{x}}_{b}(\boldsymbol{\theta})}^{\boldsymbol{\eta}} \boldsymbol{H}_{0}(\boldsymbol{\xi}, \boldsymbol{\theta}) d \boldsymbol{\xi} d \boldsymbol{\eta}$,

and from (18) it follows

$$
V_{0}\left(\boldsymbol{x}_{b}, \boldsymbol{\theta}\right)-V_{0}\left(\overline{\boldsymbol{x}}_{b}(\boldsymbol{\theta}), \boldsymbol{\theta}\right)>\lambda_{0} \frac{1}{2}\left\|\boldsymbol{x}_{b}-\overline{\boldsymbol{x}}_{b}(\boldsymbol{\theta})\right\|_{K}^{2} .
$$

Therefore, the potential fulfills the inequality

$$
\begin{aligned}
V_{p o t}\left(\boldsymbol{x}_{b}, \boldsymbol{\theta}\right) & >\lambda_{0} \frac{1}{2}\left\|\boldsymbol{x}_{b}-\overline{\boldsymbol{x}}_{b}(\boldsymbol{\theta})\right\|_{K}^{2}+V_{c}\left(\boldsymbol{x}_{b}, \boldsymbol{\theta}\right) \\
& =\lambda_{0} \frac{1}{2}\left\|\tilde{\boldsymbol{x}}_{b}\right\|_{K}^{2}+\frac{1}{2} \boldsymbol{e}\left(\boldsymbol{x}_{b}, \boldsymbol{\theta}\right)^{T} \boldsymbol{K}_{d} \boldsymbol{e}\left(\boldsymbol{x}_{b}, \boldsymbol{\theta}\right)
\end{aligned}
$$

and thus the potential function is positive semi-definite with respect to $\boldsymbol{\theta}$ and even positive definite with respect to $\boldsymbol{e}\left(\boldsymbol{x}_{b}, \boldsymbol{\theta}\right)$ and $\tilde{\boldsymbol{x}}_{b}$. From the above inequality for $V_{\text {pot }}\left(\boldsymbol{x}_{b}, \boldsymbol{\theta}\right)$ one can easily follow that the set defined by the equations $\boldsymbol{e}\left(\boldsymbol{x}_{b}, \boldsymbol{\theta}\right)=$ $\mathbf{0}, \boldsymbol{x}_{b}=\overline{\boldsymbol{x}}_{b}(\boldsymbol{\theta})$ is an isolated minimum of $V_{\text {pot }}\left(\boldsymbol{x}_{b}, \boldsymbol{\theta}\right)$.

It is also interesting to notice that the steady state equations (19)-(20) for the case of free motion correspond to the equations $\partial V_{p o t}\left(\boldsymbol{x}_{b}, \boldsymbol{\theta}\right) / \partial \boldsymbol{x}_{b}=\mathbf{0}$ and $\partial V_{p o t}\left(\boldsymbol{x}_{b}, \boldsymbol{\theta}\right) / \partial \boldsymbol{\theta}=\mathbf{0}$.

\section{Passivity}

In order to show the passivity statement from Proposition 1 , the function $V(\boldsymbol{q}, \dot{\boldsymbol{q}})$ is considered as a storage function. Therefore, its time derivative $\dot{V}(\boldsymbol{q}, \dot{\boldsymbol{q}})=\dot{V}_{k i n}(\boldsymbol{q}, \dot{\boldsymbol{q}})+$ $\dot{V}_{p o t}\left(\boldsymbol{x}_{b}, \boldsymbol{\theta}\right)$ along the solution curves of the system (1) with the controller (10-13) is computed. Due to Property 1 the time derivatives of the kinetic energy and the potential are given by

$$
\begin{aligned}
\dot{V}_{k i n}(\boldsymbol{q}, \dot{\boldsymbol{q}})= & \dot{\boldsymbol{x}}_{b}^{T}\left(-\boldsymbol{g}_{b}\left(\boldsymbol{x}_{b}, \boldsymbol{\theta}\right)-\boldsymbol{K}_{b} \boldsymbol{x}_{b}-\boldsymbol{D}_{b} \dot{\boldsymbol{x}}_{b}\right)+ \\
& \dot{\boldsymbol{\theta}}^{T}\left(-\boldsymbol{g}_{m}\left(\boldsymbol{x}_{b}, \boldsymbol{\theta}\right)+\boldsymbol{\tau}\right)+\dot{\boldsymbol{q}}^{T} \boldsymbol{\tau}_{e x t}, \\
\dot{V}_{\text {pot }}\left(\boldsymbol{x}_{b}, \boldsymbol{\theta}\right)= & \dot{\boldsymbol{x}}_{b}^{T}\left(\boldsymbol{g}_{b}\left(\boldsymbol{x}_{b}, \boldsymbol{\theta}\right)+\boldsymbol{K}_{b} \boldsymbol{x}_{b}\right)+ \\
& \dot{\boldsymbol{\theta}}^{T}\left(\boldsymbol{g}_{m}\left(\boldsymbol{x}_{b}, \boldsymbol{\theta}\right)-\frac{\mathrm{d} V_{0}\left(\overline{\boldsymbol{x}}_{b}(\boldsymbol{\theta}), \boldsymbol{\theta}\right)}{\mathrm{d} \boldsymbol{\theta}}\right) .
\end{aligned}
$$

Substituting the control law (10-13) and considering (17), leads to

$$
\dot{V}(\boldsymbol{q}, \dot{\boldsymbol{q}})=-\dot{\boldsymbol{\theta}}^{T} \boldsymbol{D}(\boldsymbol{\theta}) \dot{\boldsymbol{\theta}}-\dot{\boldsymbol{x}}_{b}^{T} \boldsymbol{D}_{b} \dot{\boldsymbol{x}}_{b}+\dot{\boldsymbol{q}}^{T} \boldsymbol{\tau}_{e x t},
$$

from which one can follow the passivity property easily.

\section{E. Stability}

For a redundant robot with $r=n$ the above analysis can be refined. In this case $V(\boldsymbol{q}, \dot{\boldsymbol{q}})$ can be considered as a Lyapunov function. Considering (8) one can see that the derivative of $\overline{\boldsymbol{x}}_{b}(\boldsymbol{\theta})$ w.r.t $\boldsymbol{\theta}$ is given by

$$
\frac{\partial \overline{\boldsymbol{x}}_{b}(\boldsymbol{\theta})}{\partial \boldsymbol{\theta}}=\boldsymbol{K}_{b}^{-1}\left(-\boldsymbol{H}_{g}\left(\boldsymbol{x}_{b}, \boldsymbol{\theta}\right)+\boldsymbol{H}_{c}\left(\boldsymbol{x}_{b}, \boldsymbol{\theta}\right)\right) .
$$

From this it follows that the Jacobian

$$
\frac{\mathrm{d} \boldsymbol{e}\left(\overline{\boldsymbol{x}}_{b}(\boldsymbol{\theta}), \boldsymbol{\theta}\right)}{\mathrm{d} \boldsymbol{\theta}}=\boldsymbol{J}_{m}\left(\overline{\boldsymbol{x}}_{b}(\boldsymbol{\theta}), \boldsymbol{\theta}\right)+\boldsymbol{J}_{b}\left(\overline{\boldsymbol{x}}_{b}(\boldsymbol{\theta}), \boldsymbol{\theta}\right) \frac{\partial \overline{\boldsymbol{x}}_{b}(\boldsymbol{\theta})}{\partial \boldsymbol{\theta}}
$$

is non-singular, if the Jacobian of the arm $\boldsymbol{J}_{m}\left(\boldsymbol{x}_{b}, \boldsymbol{\theta}\right)$ is non-singular and the base stiffness is sufficiently high. This assumption makes the potential $V_{\text {pot }}\left(\boldsymbol{x}_{b}, \boldsymbol{\theta}\right)$ positive definite w.r.t. $\tilde{\boldsymbol{x}}_{b}$ and $\boldsymbol{\theta}$. Notice that in the previous analysis it was only shown to be positive definite w.r.t. $\tilde{\boldsymbol{x}}_{b}$ and $\boldsymbol{e}\left(\boldsymbol{x}_{b}, \boldsymbol{\theta}\right)$. For the case of free motion, i.e. for $\boldsymbol{\tau}_{\text {ext }}=\mathbf{0}$, the time derivative $\dot{V}(\boldsymbol{q}, \dot{\boldsymbol{q}})$ from (23) is then negative semi-definite implying stability. Furthermore, asymptotic stability can be shown by invoking LaSalle's invariance principle [16].

Considering the redundant case it should be mentioned that, unless the controller is augmented by an additional null-space stiffness component, one can of course only expect convergence of the Cartesian error, but not asymptotical stability since $\boldsymbol{e}\left(\overline{\boldsymbol{x}}_{b}(\boldsymbol{\theta}), \boldsymbol{\theta}\right)=\mathbf{0}$ then does not define a unique joint configuration.

\section{Simulation Study}

For the evaluation of the proposed controller a simple planar system according to Fig. 2 is considered. A three DoF arm is mounted on a one DoF flexible base. The inertia of the arm and of the base is modeled by point masses. For the arm these point masses are attached to the middle of link 1 and 2 and to the endpoint of link 3 . The stiffness and damping parameters of the elastic base were chosen as $K_{b}=10000 \mathrm{Nm} / \mathrm{rad}$ and $D_{b}=$ $1400 \mathrm{Nms} / \mathrm{rad}$. This value of $D_{b}$ corresponds to a damping factor of 0.7 for the base motion when the load of the arm is neglected. All the other model parameters are depicted in Fig. 2.

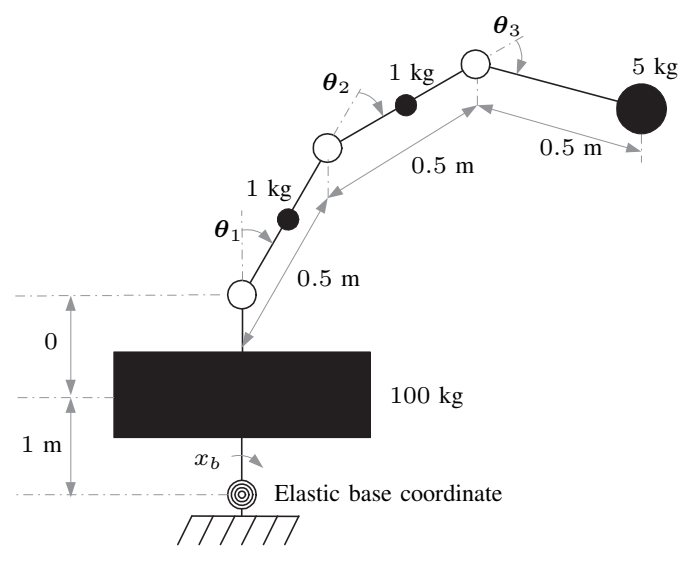

Fig. 2. Simulation Model 
As Cartesian coordinates $\boldsymbol{f}\left(\boldsymbol{x}_{b}, \boldsymbol{\theta}_{b}\right) \in \mathbb{R}^{3}$ the position of the endpoint of link 3 and the orientation of link 3 are chosen. The controller gain matrices for the desired stiffness $\boldsymbol{K}_{d}$ and the desired damping $\boldsymbol{D}_{d}$ are chosen as diagonal matrices $\boldsymbol{K}_{d}=$ $\operatorname{diag}\left(K_{d, x}, K_{d, y}, K_{d, r}\right)$ and $\boldsymbol{D}_{d}=\operatorname{diag}\left(D_{d, x}, D_{d, y}, D_{d, r}\right)$ with different entries for the translational and for the rotational coordinates. In the first simulation the position accuracy of the proposed controller is compared with a Cartesian compliance controller in which the base motion is ignored. Therefore, the desired Cartesian stiffness for the translational coordinates is chosen quite low as $K_{d, x}=K_{d, y}=100 \mathrm{~N} / \mathrm{m}$. The orientational stiffness is chosen as $K_{d, r}=50 \mathrm{Nm} / \mathrm{rad}$. The damping parameters are simply chosen as $D_{d, x}=D_{d, y}=20$ $\mathrm{Ns} / \mathrm{m}$ and $D_{d, r}=10 \mathrm{Nms} / \mathrm{rad}$.

The initial configuration (see Fig. 3) is chosen such that initially no gravity load is exerted on the elastic coordinate $x_{b}$. Consequently, the initial base deflection is zero. Starting from the initial end-effector position the desired virtual equilibrium position $\boldsymbol{x}_{0} \in \mathbb{R}^{3}$ is then moved about $0.5 \mathrm{~m}$ horizontally along the $x$-axis. This movement is generated by computing the step response of a second-order filter with transfer function $\frac{1}{s^{2}+2 s+1}$. Figure 3 also shows the joint configuration of the arm which corresponds to the steady state of the virtual equilibrium position when the base flexibility is neglected.

The desired motion in $\mathrm{x}$-direction is shown in Fig. 4. The translational error $\left.e_{t}=\sqrt{(} e_{x}^{2}+e_{y}^{2}\right)$ resulting from the proposed flexible base compliance controller (10-13) is shown in Fig. 5. The computation of $\overline{\boldsymbol{x}}_{b}(\boldsymbol{\theta})$ is done based on the

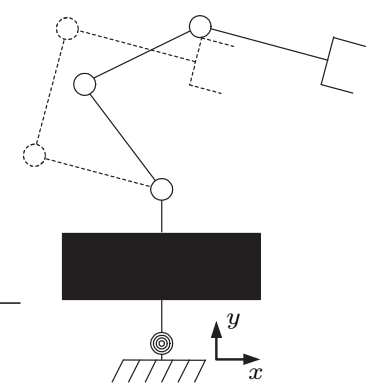

Fig. 3. Initial configuration (dashed) and desired final configuration (solid) when the base flexibility is neglected.

iteration (9) with three iteration steps. The result is compared with a simpler reference controller in which the base deflection is ignored. This reference controller can be obtained simply by setting $\overline{\boldsymbol{x}}_{b}$ in (10-13) to zero. In Fig. 5 one can see that the transient behavior of the two controllers is similar. But in steady state the neglect of the base deflection in the reference controller results in a considerable position error. This error is eliminated by the proposed controller. Since it is not obvious from Fig. 5, it should be mentioned that the error does not go to zero exactly, because $\overline{\boldsymbol{x}}_{b}$ represents an approximation (via the iteration (9)). In the present simulation with three iteration steps the remaining translational error was less than $1.810^{-5} \mathrm{~m}$ and it could be further reduced by computing a more accurate approximation of $\overline{\boldsymbol{x}}_{b}$.

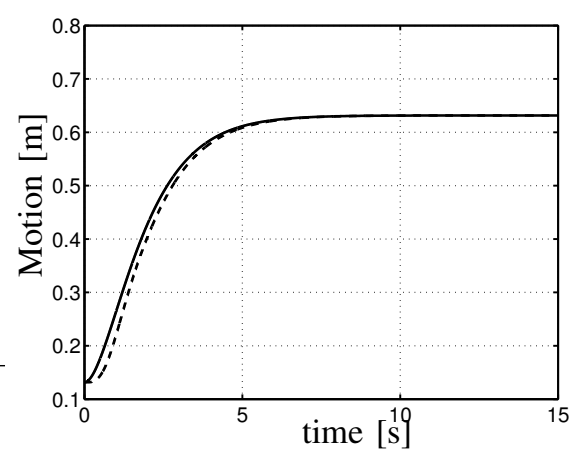

Fig. 4. Motion in $x$-direction. The solid line shows the desired motion and the dashed line the actual motion with the flexible base compliance controller.

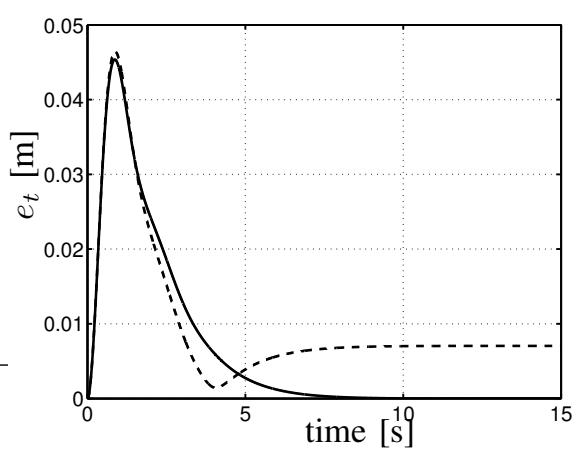

Fig. 5. Translational error $e_{t}$ for the flexible base compliance controller (solid) and the reference controller (dashed).

The same behavior can also be observed for the orientation error $e_{r}$ shown in Fig. 6. The corresponding base deflection shown in Fig. 7 is similar for both controllers. Evidently, the main amount of the steady state orientation error for the reference controller stems from the base deflection. This error is eliminated by the flexible base controller. Figure 7 shows the estimate $\overline{\boldsymbol{x}}_{b}(\boldsymbol{\theta})$ of $\boldsymbol{x}_{b}$. One can see that the three iteration steps already lead to a very good estimate in steady state.

In addition to the position accuracy, the resulting stiffness is evaluated in a second simulation. As an initial configuration the final configuration of the first simulation is chosen. Also the same stiffness and damping parameters are used. Now an external torque acts on the end-effector. The torque is chosen as a step response of a pre-filter with transfer function $\frac{1}{s^{2}+2 s+1}$ to a steady state value of $5 \mathrm{Nm}$. Therefore, in steady state the orientation error $e_{r}$ of the end-effector should reach a value of $0.1 \mathrm{rad}$ according to the desired rotational stiffness of $K_{d, r}=$ $50 \mathrm{Nm} / \mathrm{rad}$ while the translational error $e_{t}$ should be zero. The resulting orientation error is shown in Fig. 8. Additionally Fig. 9 shows the translational error. Here, the simulation was performed with three as well as with five iteration steps in the computation of $\overline{\boldsymbol{x}}_{b}(\boldsymbol{\theta})$. One can see that the resulting endeffector error (in translation), which is already quite small for three iteration steps, can be further reduced by computing a more accurate estimation of $\overline{\boldsymbol{x}}_{b}(\boldsymbol{\theta})$. 


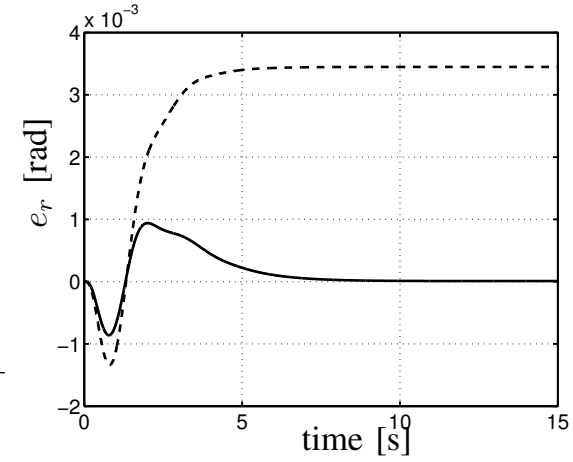

Fig. 6. Orientational error $e_{r}$ for the flexible base compliance controller (solid) and the reference controller (dashed).

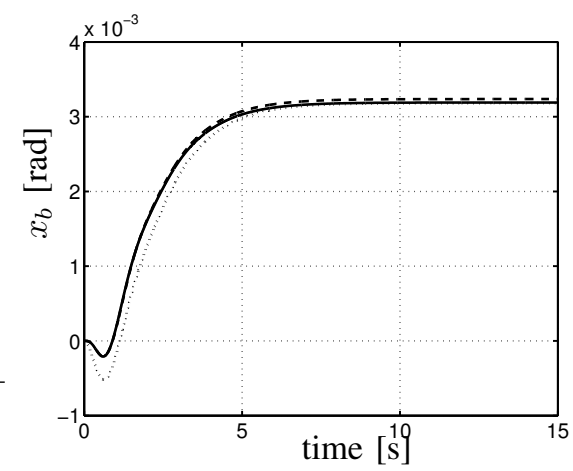

Fig. 7. Base deflection $x_{b}$ for the proposed controller (solid) and the reference controller (dashed). The dotted line shows the estimate $\bar{x}_{b}(\boldsymbol{\theta})$ of $x_{b}$.

\section{Summary}

The main contribution of this paper is the formulation of a Cartesian compliance control law for a manipulator mounted on a flexible structure. The controller does not need any measurement of the base motion, but still compensates for the static effects of the (known) base elasticity. Asymptotic stability for the case of free motion, as well as a useful passivity property for interaction tasks were shown. Finally, a planar simulation example was presented in order to evaluate the proposed controller.

\section{REFERENCES}

[1] A. Sharon and D. Hardt, "Enhancement of robot accuracy using endpoint feedback and a macro-micro manipulator system," in American Control Conference, 1984, pp. 1836-1842.

[2] D. N. Nenchev, K. Yoshida, P. Vichitkulsawat, and M. Uchiyama, "Reaction null-space control of flexible structure mounted manipulator systems," IEEE Transactions on Robotics and Automation, vol. 15, no. 6, pp. 1011-1023, 1999.

[3] J. Ueda and T. Yoshikawa, "Mode-shape compensator for improving robustness of manipulator mounted on flexible base," IEEE Transactions on Robotics and Automation, vol. 20, no. 2, pp. 256-268, 2004.

[4] I. Sharf, "Active damping of a large flexible manipulator with a shortreach robot," in American Control Conference, 1995, pp. 3329-3333.

[5] J. Lew and D. J. Trudnowski, "Vibration control of a micro/macromanipulator system," IEEE Control Systems Magazine, pp. 26-31, 1996.

[6] D. W. Cannon, D. P. Magee, W. J. Book, and J. Y. Lew, "Experimental study on micro/macro manipulator vibration control," in IEEE International Conference on Robotics and Automation, 1996, pp. 2549-2554.

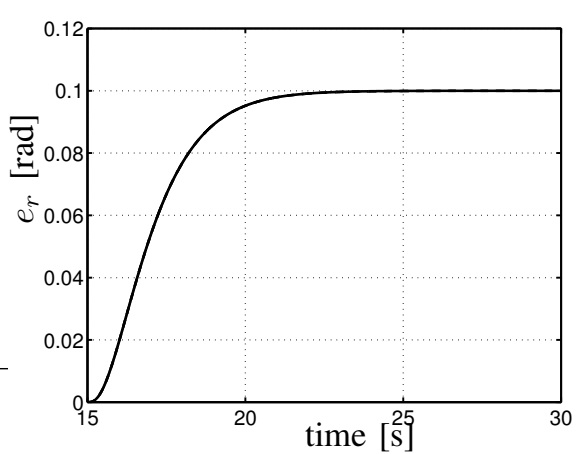

Fig. 8. Orientational error $e_{r}$ for stiffness evaluation

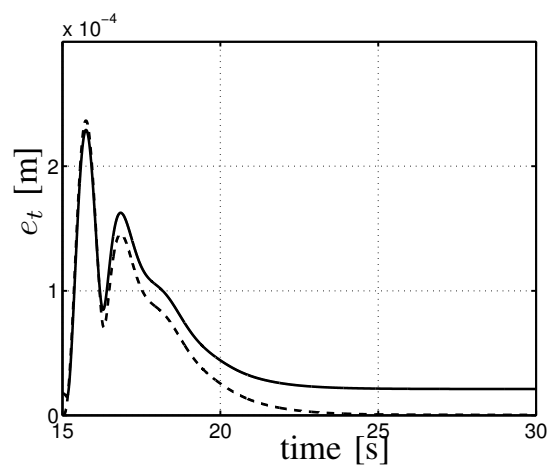

Fig. 9. Translational error $e_{t}$ for the stiffness evaluation. The solid and dashed line show the results with three and five iteration steps, respectively.

[7] J. Lew and S.-M. Moon, "A simple active damping control for compliant base manipulators," IEEE/ASME Transactions on Mechatronics, vol. 6, no. 3, pp. 305-310, 2001.

[8] Ch. Ott, A. Albu-Schäffer, A. Kugi, S. Stramigioli, and G. Hirzinger, "A passivity based cartesian impedance controller - part I: Torque feedback and gravity compensation," in IEEE International Conference on Robotics and Automation, 2004, pp. 2659-2665.

[9] A. Albu-Schäffer, Ch. Ott, and G. Hirzinger, "A passivity based cartesian impedance controller - part II: Full state feedback, impedance design and experiments," in IEEE International Conference on Robotics and Automation, 2004, pp. 2666-2672.

[10] — "Passivity based cartesian impedance control for flexible joint manipulators," in IFAC Symposium on Nonlinear Control Systems, 2004.

[11] _ "Constructive energy shaping based impedance control for a class of underactuated euler-lagrange systems," in IEEE International Conference on Robotics and Automation, 2005, pp. 1399-1405.

[12] Ch. Ott, A. Albu-Schäffer, A. Kugi, S. Stramigioli, and G. Hirzinger, "Cartesian impedance control of flexible joint robots: A passivity based approach," at-Automatisierungstechnik, pp. 378-388, 82005.

[13] Ch. Ott, "Cartesian impedance control of flexible joint manipulators," Ph.D. dissertation, Saarland University, 2005.

[14] C. de Wit, B. Siciliano, and G. Bastin, Eds., Theory of Robot Control, ser. Communications and Control Engineering. Springer-Verlag, 1996.

[15] K.-S. Chang and O. Khatib, "Manipulator control at kinematic singularities: A dynamically consistent strategy," in IEEE/RSJ International Conference on Intelligent Robots and Systems, 1995, pp. 84-88.

[16] M. Vidyasagar, Nonlinear Systems Analysis, 2nd ed. Prentice Hall, 1993.

[17] S.-F. Chen and I. Kao, "Conservative congruence transformation for joint and cartesian stiffness matrices of robotic hands and fingers," The International Journal of Robotics Research, vol. 19, no. 9, pp. 835-847, 2000.

[18] A. van der Schaft, $L_{2}$-Gain and Passivity Techniques in Nonlinear Control, 2nd ed. Springer-Verlag, 2000. 